\title{
The cost of children: May the collective approach to household behavior help?
}

\author{
François Bourguignon \\ DELTA/E.N.S., 48 Bd. Jourdan, F-75014 Paris, France (e-mail: bourg@delta.ens.fr)
}

Received: 29 August 1997/Accepted: 26 November 1998

\begin{abstract}
The collective approach to household consumption behavior tries to infer from variables supposed to affect the general bargaining position of household members information on the allocation of consumptions goods and tasks among them. This paper investigates the extension of previous work to the case where children may be considered as a public consumption good by the two adult members of a household. The main question being asked is whether it is possible to retrieve from the aggregate consumption behaviour of the household and the relative earnings of the parents information on the allocation of goods between them and children. This alternative approach to the estimation of the 'cost of children' is contrasted with the conventional approach based on a 'unitary' representation of and demographic separability assumptions on household consumption behaviour.
\end{abstract}

JEL classification: D10, J13, J15

Key words: Consumption behaviour, intra-household allocation, cost of children

\section{Introduction}

Measuring the cost of children has long been on the agenda of economists. Yet it is still largely an unsolved problem. That children do not have the same

This paper is an extension of the presidential address given at the $10^{\text {th }}$ Congress of the European Society of Population Economics, Lisbon, 1995. Since then, it was also presented at various seminars. I thank participants of these seminars as well as two referees for their comments. I also thank Oliver Doni for a particularly scrupulous reading of an earlier version. Of course, all errors remain mine. Responsible editor: Klaus F. Zimmermann. 
consumption needs as adults and that a correction should be made for demographic composition when comparing the welfare of different households is rather obvious. Accordingly, several methods have been proposed which yield equivalence scales allowing the comparison of the welfare of families with different compositions by simply deflating their income with a suitable demographic index. Some scales are based on mostly a priori judgements. They then usually draw on nutritionists' studies of the cost of feeding people of different ages with a suitable adjustment for non-food expenditures. Others rely on the observed consumption behavior of households with different demographic compositions and the assumption that comparable structures of consumption should somehow correspond to comparable levels of welfare. It is now, however, firmly established that the welfare theoretical basis of all these methods is weak, not to say non-existing (See in particular Pollak and Wales (1979), Fischer (1987) and Blundell and Lewbel (1991)).

What is wrong with standard welfare comparisons across households of different types is essentially that welfare is defined as a family attribute when it can logically be only an individual attribute. In the words of Deaton and Muellbauer (1986): "Ultimately, welfare comparisons must be based on the welfare levels of individuals, not of households..." (My italics). This means that the theoretical background necessary for comparing households with different compositions must explicitly be the process of intra-household allocation of goods and services among family members, welfare comparisons being then made at the level of every individual in the family and what he/she actually receives. This is in striking contrast to that part of the literature on the cost of children which is based on a 'unitary' view of the household, where a single rational decision-maker makes decisions on essentially aggregate consumption goods. The only exception to this may be various unitary models inspired by Rothbarth's idea of identifying the intra-household allocation process between adults and children by considering goods exclusively consumed by adults under the debatable assumption that preferences for these goods are identical within households with different compositions.

All this seems to lead to a rather pessimistic view of the possibility of ever grounding empirical inter-household welfare comparisons in a rigorous spending behavior framework. To quote Deaton and Muellbauer again "We doubt that expenditure data in anything like their traditional form can tell us very much about the relative welfare levels of adults and children." They add, "One possible assumption would be that everyone in the household shares the same level of welfare, and this would enable comparisons of welfare or inequality with individuals being the basis of analysis. However, there are cases in which such an assumption would clearly be inappropriate..."

The question I consider in this paper is whether the recent theoretical and empirical developments in the analysis of intra-family allocation of goods under the general heading of the 'collective' approach to household behavior may permit going over some of the previous difficulties of welfare comparisons among families with different compositions. (For a general presentation of this approach see Bourguignon and Chiappori (1992).) Being explicitly grounded on individual, possibly altruistic, preferences, and individual consumption, this approach should in effect lead to welfare comparisons explicitly based on individuals rather than on loosely defined family utility functions. For the moment this strand of literature has insisted on positive behavior and the mere possibility of testing the "collective" hypothesis, that is the Pareto 
efficiency of family decisions, versus the unitary model and possibly other models of family decision making. Not much has been done, however, to really identify the allocation of expenditures among household members including children, and to derive welfare implications from that identification.

This is precisely what I intend to analyze in this paper by generalizing previous results obtained in Browning et al. (1994) and Bourguignon et al. (1996). I will show in particular that the collective approach allows recovering, up to a constant, the allocation of total private consumption expenditures among various adults and children in a family without any assumption on the comparability of consumption behavior among households with different compositions being necessary. Dispensing with this assumption of 'demographic separability' to identify the intra-household allocation of goods might represent significant progress in the measurement of the cost of children, even though this identification is made up to a constant and is thus only partial.

The presentation is organized in the three following parts. I will first briefly summarize the conventional approach to the measurement of the cost of children, its basic assumptions and its limitations. I will then offer a short presentation of the collective approach to household behavior as it presently stands, using in the background several recent papers in common with M. Browning, P.-A. Chiappori and V. Lechêne. I will finally investigate extensions which permit handling the issue of the cost of children within the 'collective' model.

It must be stressed that this paper is essentially theoretical, even though it deals with what is largely an empirical issue. At the present stage, I simply hope to convince readers that the collective approach to household behavior suggests theoretical answers on the issue of the cost of children which are worth serious new empirical investigation. I should also add that I will refer throughout this paper to the notion of the cost of children as essentially the budget devoted in a family to the consumption of goods and services by children and therefore made unavailable to parents. I shall not include in it the utility -or disutility- that parents may derive directly from having children and, as pointed out repeatedly in the literature since Pollak and Wales (1979), cannot be recovered from the sole observation of spending behavior. In view of the little that we know about it, I do not think this is a severe restriction. (See Kapteyn (1994) on the use of subjective welfare scales in the measurement of the cost of children.)

\section{The conventional approach to the measurement of the cost of children}

The point is not to summarize here the huge literature on the subject but only to stress the basic assumptions and methodological choices behind common practice in this area and their limitations (for a very recent survey, see van Praag and Warnaar (1997)).

In view of the introduction to this paper, two types of approach must be distinguished because of their different emphases on the intra-family allocation of goods. Methods in the Rothbarth tradition seek to identify which part of the budget of a family goes to children by analyzing differences in the consumption of an "adult good" in families with and without children. On the contrary, more standard and conventional methods see the family as a black box and base the estimation of equivalence scales on the observation of dif- 
ferent consumption behavior in families with different demographic composition. I will focus here on the first approach because of the close connection of the collective model with it. However, the possible relationship between this model and the more conventional Barten approach to equivalence measurement will also be considered.

\subsection{The Rothbarth tradition}

The best recent references for this approach are Lazear and Michael (1986), Deaton and Muellbauer (1986), Deaton et al. (1989), and Gronau (1991). I am giving here only the essence of the economic argument and of the methodology for estimating the cost of children.

Assume there are only private consumption goods and that it is possible to partition them into adult goods, $x^{a}$, children goods, $x^{c}$, and mixed goods, $x^{m}$, of which $x^{m a}$ is consumed by adults and $x^{m c}$ by children. Assume also that the preferences of a household with one child may be represented by the following utility function, weakly separable in $\left(x^{a}, x^{m a}\right)$ :

$$
U^{c}\left[\varphi\left(x^{m a}, x^{a}\right), x^{c}, x^{m c}\right]
$$

where both $U^{c}()$ and $\varphi()$ have the usual properties with respect to their arguments (increasing and quasi-concave). Assume finally that the preferences of a family without children is given as a particular case of (1), that is:

$$
U^{a}\left[\varphi\left(x^{m a}, x^{a}\right)\right]
$$

The demographic separability assumption corresponding to (1) and (2) is equivalent to assuming that the marginal rates of substitution between goods consumed by adults is the same - e.g. one bottle of Dom Perignon against half a pretty dress - whether they have children or not, and if they have children, whatever is consumed by the children. This certainly is a strong restriction, but nothing is possible within the conventional 'unitary' framework for household consumption choices without an assumption of this type.

The maximization of (2) under the usual budget constraint leads to the following demand functions:

$$
p_{i}^{a} x_{i}^{a}=D_{i}^{a}\left(p^{a}, p^{m} ; y\right)
$$

where $y$ is the consumption budget and $p^{j}$ are vectors of prices associated to goods $j(=a, m, c)$. The indirect utility associated to (2) may be written as:

$$
V^{a}\left(p^{a}, p^{m} ; y\right)
$$

Consider now the family with one child and preferences (1). Two-stage budgeting leads to the following demand function for adult goods:

$$
p_{i}^{a} x_{i}^{a}=D_{i}^{a}\left(p^{a}, p^{m} ; y^{a}\right)
$$

where $y^{a}$ is that part of the total budget, $y$, which is allocated to the consumption of adults. 
We now have all the elements to measure which part of the budget goes to the child in a family of type c. Suppose we observe: a) the demand function for adult goods in families without children, $D_{i}^{a}(\quad) ; \mathrm{b}$ ) the expenditures of one (or several) adult goods in families with a child, say $\operatorname{good}_{1} p_{1}^{a} x_{1}^{a}$; c) all families face the same price system (the usual assumption for cross-sectional data). Then two consequences follow:

(i) the part of the budget going to the child is given by:

$$
y^{c}=y-M\left(p_{1}^{a}, x_{1}^{a}\right)
$$

where $M()$ is the inverse of the demand function (3) with respect to the budget variable, $y$. by:

(ii) A measure of the welfare of adults in families with a child is given

$$
V^{a}\left[p^{a}, p^{m} ; M\left(p_{1}^{a}, x_{1}^{a}\right)\right]
$$

We could go into more detail and ask now what happens when several adult goods are observed simultaneously, when the number of children is variable, when there are price-variations across families, etc. But as the emphasis here is on the nature of the assumptions that are made and the methodology that is used, there is no need to consider these complications. The important conclusion to be drawn from the preceding simple argument is that observing the consumption of at least one adult good and the total income in all households in a population is sufficient to determine, on one hand, the distribution of expenditures among adults and, on the other hand, that among children.

In a world where households would be either two adults with no child or two adults with a child, this would indeed be sufficient to handle distributional and welfare issues truly at the individual level with an explicit distinction between adults and children, rather than at the ill-defined household level. Note, however, that the preceding framework does not yield any information about the welfare of children in comparison to that of adults. Yet such an assumption would be necessary if one were to compare the total welfare of families with different numbers of children. From that point of view, point (ii) above about the welfare of adults in families with a child is not really very useful, unless one makes the additional assumption that all individuals in a household necessarily enjoy the same level of welfare. In that case, two couples with the same consumption of adult goods should have the same level of welfare, whether they have a child or not.

In summary, the Rothbarth type approach to individual welfare measurement within households permits identifying the cost of children through: a) a separability assumption on adult goods - which may be easily tested where more than one adult good is observed or prices are variable; b) the estimation of the demand for adult goods in a reference group. Here, this group was adults without children, but things are more complex when one considers simultaneously various family sizes, the age of the children, etc. An additional assumption worth emphasizing is that all goods are private. The two-stage budgeting operation leading to (5) above would not be possible in presence of public goods. 


\subsection{The Engel-Barten-Gorman tradition}

A model much more commonly used to measure the cost of children, and more generally to compare households with different demographic composition is that of Barten (1964). It is more elaborate than the preceding one in the sense that it does not require the existence of goods which are exclusively consumed by adults. It is also based on the idea, originally attributable to Engel, that households with similar consumption patterns with respect to some goods must share the same level of welfare.

Within the same framework as described above, that is two groups of families, couples without children and couples with a child, we now assume that no distinction is possible between goods consumed by adults or children; all goods the expenditures of which are statistically observed are consumed simultaneously by both adults and children. Denote the utility of adults in families without children by:

$$
u^{a}=U\left(x_{1}, x_{2}, \ldots, x_{n}\right),
$$

where $U()$ is a conventional ordinal utility function representing the preferences of adult households, and assume that the utility function for families with a child is given by:

$$
u^{c}=U\left(\frac{x_{1}}{q_{1}}, \frac{x_{2}}{q_{2}}, \ldots, \frac{x_{n}}{q_{n}}\right) .
$$

The parameters $q_{i}$ may be considered as equivalence scales specific to each good. Thus $q_{i}$ would be equal to unity for adult goods, if such goods could be observed, and $q_{i}$ would be greater than unity for goods consumed by both adults and children. These specific equivalence scales may be estimated by comparing the observed spending behavior of the two types of families.

As it is well known, the maximization of (7) under the usual budget constraint is equivalent to that of (6) after the following transformation:

$$
x_{i}^{*}=x_{i} / q_{i} \quad p_{i}^{*}=p_{i} q_{i} .
$$

The cost functions associated to a level of utility, $u$, are in each case:

$$
c(u, p) \text { and } c(u, p . q)
$$

and the cost of a child is then defined, in an apparently 'natural' way, as the difference:

$$
y^{c}=c(u, p . q)-c(u, p)
$$

which depends in general on the level of utility of the household of reference, $u$, and the vector of prices, $p$. The amount $y^{c}$ is simply the additional budget needed for making a couple with a child as well-off as a childless couple with utility level $u$.

In summary, the identification of the cost of children is obtained here through the following two assumptions: a) there exists a set of good-specific equivalence scales such that the consumption preferences of a family with one 
child can be made strictly identical to that a family without children; b) two families with identical 'equivalized' consumptions, $x^{*}$, have the same welfare, whether they have a child or not.

There is some similarity between this and the Rothbarth model in the sense that two families consuming the same quantities of goods that are somehow made 'equivalent' to adult goods, or indifferent bundles of these goods, are supposed to have the same level of welfare. The difference with the Rothbarth approach is that this equivalence with adult goods is not explicitly defined and, oddly enough, is not independent from the consumption of children. This makes the role of intra-household allocation issues in this model very unclear and its welfare implications, as well as the corresponding evaluation of the cost of children, essentially ambiguous.

We examine in the rest of this paper whether the 'collective' approach to household consumption and its emphasis on intra-household allocation permit a more direct evaluation of the cost of children. Before considering the issue of the cost of children and intra-household allocation of goods in families with children, we first recall the main lines of this approach in the case of two adults only.

\section{The collective approach to household consumption behavior}

\subsection{The general framework}

Stepping back a little, we now consider households made up of two adults, $A$ and $B$, with preferences defined on the vector of goods they consume. To simplify, we assume all goods are private. At this stage, a first difference with the unitary model used above for the childless family is that we now consider explicitly two utility functions:

$$
U^{A}\left(x_{1}^{A}, x_{2}^{A}, \ldots, x_{n}^{A}\right) \quad U^{B}\left(x_{1}^{B}, x_{2}^{B}, \ldots, x_{n}^{B}\right)
$$

which describe the preferences of the two household members. It does not change the analysis to consider altruistic agents in the sense of Becker's notion of "caring", that is with utility functions which depend on the other's vector of consumption with the following symmetric separability property:

$$
V^{A}\left[U^{A}\left(x^{A}\right), U^{B}\left(x^{B}\right)\right] \quad V^{B}\left[U^{A}\left(x^{A}\right), U^{B}\left(x^{B}\right)\right],
$$

where $V^{A}()$ and $V^{B}()$ are increasing and quasi-concave. We momentarily rule out public goods and/or consumption externalities such that the preceding functions would depend almost without restriction on the two vectors $x^{A}$ and $x^{B}$.

We assume in all that follows that labor supply is fixed at full-time. (Laborsupply issues linked to the collective framework are analyzed in detail in Chiappori $(1988,1992)$.) Thus, each member earns an exogenous income $y_{i}$, $i=A, B$, whereas they jointly receive a non-labor income equal to $y_{0}$. We also assume that there is no saving and that all households face the same price system $p$, so that this vector may be simply normalized to $1, p=e=$ 
$(1, \ldots, 1)$. The budget constraint faced by the couple $(A, B)$ thus is:

$$
e^{\prime}\left(x^{A}+x^{B}\right)=y=y_{1}+y_{2}+y_{0} .
$$

The collective model of household behavior is based on the assumption that the allocation of goods within the household is made on an undefined cooperative process leading to a Pareto-efficient allocation. It may easily be shown that the set of Pareto-optima obtained with the 'caring' utility functions, $V^{j}()$, is included in the set of optima resulting from the egocentric functions, $U^{j}()$. Without any loss of generality, we may thus define the Paretoefficiency of the household allocation process as follows.

Pareto-efficiency. There exists for any household a positive scalar $\mu$, such that the intra-family allocation of goods satisfies:

$$
\operatorname{Max}_{x^{A}, x^{B}} U^{A}\left(x^{A}\right)+\mu U^{B}\left(x^{B}\right) / e\left[x^{A}+x^{B}\right]=y .
$$

But nothing is known a priori of that scalar $\mu$, which represent at the same time the bargaining power and altruistic attitudes of family members. This scalar may thus be any function of all the exogenous characteristics of the household, including in the present case the full-time earnings of its members and unearned income $\left(y_{A}, y_{B}, y_{0}\right)$. For instance, with an interpretation of the objective function (8) drawn from bargaining theory, it may be thought that the member with the highest earnings has more power than his/her partner, this being less true in a couple where unearned income is very large. But, of course, making $\mu$ a function of all characteristics of the household is consistent with many theories of the household decision process. We do not need more for the moment.

The objective function (8) is not necessarily in contradiction with what would be implied by the conventional unitary model of the household. In that model, an undefined agent would be maximizing a 'household' utility function the arguments of which would be $x^{A}$ and $x^{B}$, or more usually, the aggregate consumption $x^{A}+x^{B}$. There are, however, two important differences. The first is that, in the present case, this household utility function depends, through the weighting factor $\mu$, on the total income of the household and its various income sources. (Without the assumption of common unitary prices for all households, the arguments of $\mu$ should also include the whole vector of prices of consumption goods.) The second is the (additive) separability of $x^{A}$ and $x^{B}$ in the objective function in (8) which leads to the following concept of a sharing rule between the two family members.

Sharing rule. The first theorem of welfare economics implies that there exists a function $\alpha\left(y_{A}, y_{B}, y_{0}\right)$, such that the social objective (8) may be decentralized in the following way:

$$
\left\{\begin{array}{l}
\operatorname{Max}_{x^{A}} U_{A}\left(x^{A}\right) / e x^{A}=\alpha \\
\operatorname{Max}_{x^{B}} U_{B}\left(x^{B}\right) / e x^{B}=y-\alpha
\end{array}\right.
$$

$\alpha$ is that part of the total budget $y$ which goes to member A and we shall refer 
to it as the sharing rule in what follows. As it makes the two objectives (8) and (9) equivalent, it clearly depends on the way the weighting factor $\mu()$ is itself influenced by the various sources of incomes, as well as on total income and the shape of individual utility functions.

Empirically, the difficulty is that one generally observes the aggregate consumption $\left(x^{A}+x^{B}\right)$, rather than the individual vectors $x^{A}$ and $x^{B}$. The problem is thus to know whether the preceding assumptions generate restrictions on observed aggregate consumption functions so that the collective model is observationally distinguishable from the unitary model analyzed in the preceding section and from more other models of household consumption.

Let us consider first the case where no good is assignable in the sense that it is impossible to say whether it is consumed and in what proportion by member $A$ or $B$. Household consumption of any good $i$ is then the sum of the consumption of that good by members $A$ and $B$ and may be written as the following function of the exogenous characteristics of the household, and in particular the three sources of income:

$$
x_{i}\left(y_{A}, y_{B}, y_{0}\right)=F_{i}^{A}\left[\alpha\left(y_{A}, y_{B}, y_{0}\right)\right]+F_{i}^{B}\left[y-\alpha\left(y_{A}, y_{B}, y_{0}\right)\right] \quad \forall i,
$$

where $F_{i}^{j}()(j=A, B)$ are the demand functions derived from (9), i.e. in the present case the individual Engel curves.

The question we now ask is whether (10) implies some testable restrictions on the observed aggregate consumption behavior of households. Because the function $\alpha($ ) appears in the two terms in the right-hand side of that equation, the answer is yes and here are some of them - a complete exposition may be found in Bourguignon et al. (1996).

(i) Absence of income pooling. Taking derivatives of (10) it readily appears that it is in general the case that, for any good $i$ :

$$
\frac{\partial x_{i}}{\partial y_{A}} \neq \frac{\partial x_{i}}{\partial y_{B}} \neq \frac{\partial x_{i}}{\partial y_{0}} .
$$

In other words, unlike in the unitary model, marginal propensities to consume depend on the sources of income.

(ii) Proportionality of marginal propensities to consume. However, these marginal propensities to consume out of different sources must be proportional to each other across the various goods. This is because they are all derived from the sharing rule function which appears in all consumption functions:

$$
\frac{\frac{\partial x_{i}}{\partial y_{k}}}{\frac{\partial x_{i}}{\partial y_{l}}}=\frac{\frac{\partial x_{j}}{\partial y_{k}}}{\frac{\partial x_{j}}{\partial y_{l}}} \quad \text { for all }(i, j) \in(1,2, \ldots) \text { and } \quad(k, l) \in(A, B, 0) .
$$

\subsection{Recovering the sharing rule and individual preferences}

An important property from the point of view taken in this paper is whether it is possible to recover from the observation of the aggregate consumption 
behavior of the household, that is the generalized consumption functions $x_{i}\left(y_{A}, y_{B}, y_{0}\right)$, the sharing rule $\alpha\left(y_{A}, y_{B}, y_{0}\right)$. If this were possible, then observing the way household consumption depends on the various sources of income would at the same time reveal, under the assumption that property (12) above holds, the intra-family allocation of the consumption budget. We show in what follows some simple cases where it is indeed possible to recover from observed consumption behavior the function $\alpha\left(y_{A}, y_{B}, y_{0}\right)$ up to a constant.

(i) Observing one exclusive good permits retrieving the sharing rule up to a transformation. Suppose we observe the consumption of good 1, which we know is consumed exclusively by member $A$. From (10), we have then:

$$
x_{1}\left(y_{A}, y_{B}, y_{0}\right)=F_{1}^{A}\left[\alpha\left(y_{A}, y_{B}, y_{0}\right)\right]
$$

Observing how the consumption of good 1 depends on $\left(y_{A}, y_{B}, y_{0}\right)$ thus gives direct information on how much of the total budget member $\mathrm{A}$ receives, that is $\alpha()$. However, since $F_{1}^{A}()$ is not known, $\alpha$ can be retrieved only up to some transformation, unless some extra restriction is imposed. For instance, we might want to impose a 'fair' sharing rule satisfying:

$$
\alpha\left(z, z, y_{0}\right)=z+y_{0} / 2 \text { for all } z \text { and } y_{0} \text {. }
$$

Restricting the estimation in a sample of observations to households where the two members have the same earnings would permit identifying $F_{1}^{A}()$ and then fully recovering the sharing rule $\alpha()$. This was the route taken in Browning et al. (1994). But it is possible to do better.

(ii) Observing one exclusive good and one ordinary good. Suppose now that, together with the preceding exclusive good, we observe in a household budget survey the total expenditures on a good consumed by the two household members in some unobserved proportions, i.e. an 'ordinary' good. With the previous notations, we now have the following restrictions on goods 1 and 2:

$$
\begin{aligned}
& x_{1}\left(y_{A}, y_{B}, y_{0}\right)=F_{1}^{A}[\alpha] \\
& x_{2}\left(y_{A}, y_{B}, y_{0}\right)=F_{2}^{A}[\alpha]+F_{2}^{B}[y-\alpha],
\end{aligned}
$$

where, for ease of notation, we omitted the arguments of the sharing rule function, $\alpha$.

Inverting the first equation yields $\alpha=a\left(x_{1}\right)$ where $a()$ is the inverse of the function $F_{1}^{A}()$. Substituting in the second equation of (13) leads to the following expression of the ' $x_{1}$-conditional' demand for good 2 , that is the demand for good 2 expressed as a function of the consumption of good 1 and total income: ${ }^{2}$

$$
X_{2}=\theta_{2}\left(x_{1}, y\right)=F_{2}^{A}\left[a\left(x_{1}\right)\right]+F_{2}^{B}\left[y-a\left(x_{1}\right)\right]
$$

which depends on the observed consumption of good 1 and the total budget of 
the household. Differentiate $\theta_{2}$ with respect to $y$ conditionally on $x_{1}$ :

$$
\frac{\partial \theta_{2}}{\partial y}=F_{2}^{B^{\prime}}\left[y-a\left(x_{1}\right)\right] .
$$

Differentiate again, first with respect to $y$, and then with respect to $x_{1}$. Take the ratio of these two partial derivatives to obtain:

$$
a^{\prime}\left(x_{1}\right)=-\left(\partial^{2} \theta_{2} / \partial x_{1} \partial y\right) /\left(\partial^{2} \theta_{2} / \partial y^{2}\right)
$$

This means that the function $a\left(x_{1}\right)$ and therefore the sharing rule $\alpha\left(y_{A}, y_{B}, y_{0}\right)=a\left[x_{1}\left(y_{A}, y_{B}, y_{0}\right)\right]$ are identified up to a constant from the observation of the consumption behavior of good 1 and the $x_{1}$-conditional consumption behavior of good $2 .^{3}$ It also follows that the three individual Engel $F_{i}^{j}()$ curves may also be recovered from the data up to a constant.

The sharing rule may be identified up to a constant in still more general situations. In Bourguignon et al. (1996), we show in particular that there is no need to observe an exclusive good. Observing the aggregate household consumption behavior for three 'ordinary' goods is generally sufficient to recover the sharing rule and the derivatives of all individual Engel curves. As in the preceding case, however, it is important to stress that identification is based on 2nd order properties of observed aggregate household behavior. In other words, the sharing rule consistent with observed data depends not only on the observed marginal propensities to consume out of the various income sources, but also on the way these marginal propensities change with them.

\subsection{Implications}

These results are rather remarkable. Under the assumption that individual preferences are independent from supposedly exogenous earnings, observing how aggregate household consumption depends on the various sources of income in the household permits identifying, up to a constant, how the consumption budget is allocated between household members.

More precisely, identification proceeds as follows: (a) We observe the consumption behavior of couples where both partners work full-time with earnings respectively equal to $y_{A}$ and $y_{B}$; (b) We test the income pooling hypothesis that aggregate consumption depends only on aggregate income; (c) If it is rejected, we test whether the marginal propensities to consume with respect to the various components of aggregate income are proportional across goods; (d) Observing the marginal propensities to consume with respect to the various components of aggregate income for three ordinary goods, or for one exclusive and one ordinary good permits to recover the sharing rule as well as individual Engel curves up to a constant.

The knowledge of such a sharing rule permits inferring the effect on individual welfare of any variation in individual earnings and unearned income. The intra-family allocation of the consumption budget is thus known in variational terms. Identifying fully the sharing rule can only be done by resorting to some arbitrary allocation for some reference couple. This is certainly less demanding than the fair rule (14). 
The analogy with the Rothbarth method is quite clear in the two examples used above. In both cases, observing an exclusive good permits inferring something on the way the consumption budget is allocated between family members. In the Rothbarth model, this would be done by considering the consumption behavior of a sample of bachelors made up of type $A$ persons. By inverting the corresponding Engel curve, one would then obtain the budget allocated to member $A$ and, by accounting identity, that allocated to member $B$. The big difference in the present model is that we do not need to refer to what would be the behavior of member $A$ if he/she were a bachelor and to make the debatable assumption that his/her preferences are the same whether he lives alone or with member $B$. Observing how the aggregate consumption of an additional good depends also on the various income sources in the household is sufficient to get the share of the budget allocated to members $A$ and $B$, up to a constant.

This constant could presumably be identified if indeed it were supposed that there is some analogy between the consumption behavior of individuals $A$ when they are single and when they live with individuals $B$. The difference with the identification of equivalence scales seen in the preceding section is that such an assumption must be made here only for one specific individual $A$, or rather an individual $A$ with some given income, whereas it is generally made for all individuals $A$ in the equivalence scale approach. Doing so in the present context would simply be equivalent to deriving the full Engel curves $F_{1}^{A}()$ and $F_{2}^{A}()$ from the observation of the consumption behavior of single individuals A. It would thus provide a simple way of testing the behavioral identity across demographic groups - i.e. demographic separability- or to enrich the collective model, for instance by including in it the consumption of public goods. We do not investigate this possibility here. Instead, we try to generalize the preceding analysis to handle a three-way allocation involving children.

Before turning to this, some limitations of the framework which was just presented must be stressed. Of particular importance for the whole argument is the supposed exogeneity of earnings and (full-time) labor-supply. All of the identification process which has just been described is based on the assumption that individual earnings have no influence on individual preferences among consumption goods. If differences in earnings actually reflect variations in labor-supply, then the preceding estimation procedure must be modified. In particular, comparisons of consumption and labor-supply behavior across single and married individuals will likely become necessary. (Such a comparison is made in Browning et al. (1994) and leads to the conclusion that the exogeneity of earnings in the intra-family allocation of consumption goods cannot be rejected.)

Another important limitation of the preceding framework is the absence of true consumption externalities between the two household members, or, equivalently, the absence of public goods. In theory, it should be possible to control for such public goods if they can be easily identified - housing for instance. This was done in previous work -see Bourguignon et al. (1993). But it is not always possible to do so, the most obvious example being the expenditures made on children, who following Weiss and Willis (1985) and Folbre (1994) may indeed be considered as 'public goods' for the parents. We now turn precisely to this case and show that, under some additional assumptions, the preceding identification of a household 'sharing rule' may be usefully generalized. 


\section{Collective approach with public goods and/or children}

To the extent that children may be considered as public goods, we shall not explicitly distinguish them from other public goods in what follows. Extensions in that direction could easily be thought of.

To take children into account, let us assume that the utility of the two parents takes the following form:

$$
\begin{aligned}
& V^{A}\left[U^{A}\left(x^{A}\right), U^{B}\left(x^{B}\right), U^{C}\left(x^{C}\right)\right] \\
& V^{B}\left[U^{A}\left(x^{A}\right), U^{B}\left(x^{B}\right), U^{C}\left(x^{C}\right)\right],
\end{aligned}
$$

where $x^{c}$ represents the vector of goods consumed by children and $U^{C}()$ the utility their consumption brings to their parents. This specification may be seen as an extension of the "caring" hypothesis made in the preceding section and seems a natural way of representing the altruistic behavior of parents with respect to their children. The fact that the same function $U^{C}()$ appears in the utility functions of both members means that both parents have the same preferences among the goods consumed by their children - they are willing to trade the same number of candy bars for a book - but may not value equally their total welfare. The rate of substitution between $U^{A}()$ and $U^{C}()$ in $V^{A}()$ may, for example, be larger than that between $U^{B}()$ and $U^{C}()$ in $V^{B}()$. Thus, the preceding formulation permits capturing part of the asymmetry in the preferences of the two adults with respect to expenditures on children.

If one admits the above restrictions on utilities, then the results in the previous section may be easily generalized. As before, Pareto efficiency of the allocation of consumption means that there exists a scalar $\mu$ such that the household maximizes:

$$
V^{A}\left[U^{A}\left(x^{A}\right), U^{B}\left(x^{C}\right), U^{C}\left(x^{C}\right)\right]+\mu V^{B}\left[U^{A}\left(x^{A}\right), U^{B}\left(x^{B}\right), U^{C}\left(x^{C}\right)\right]
$$

subject to:

$$
e^{\prime}\left(x^{A}+x^{B}+x^{C}\right)=y=y_{A}+y_{B}+y_{0}
$$

It may be seen that the present model is not a generalization of the preceding one in the sense that a new agent -i.e. the children- enters the intra-household allocation game. Children in the present model have no decision power and the structure and amount of expenditures on them result from the cooperative game played by the two parents. As mentioned above, they might as well be considered as any other public good consumed simultaneously by the two adult members of the couple. Doing so and ignoring the decision power of children would certainly be wrong beyond the age at which the alternative does exist for them to separate from their family but seems a sensible assumption below that limit.

As in the preceding section $\mu$ is given and depends on the household's exogenous characteristics, that is, here its various sources of income, under the assumption of exogenous earnings and full-time labor supply. ${ }^{4}$ However, the decentralized version of the preceding program, and the corresponding sharing rule must now be modified. The decentralization of decisions in (18) leads 
to defining two partial budgets $\alpha$ and $\beta$ such that the optimal allocation of goods between the private consumption of $A$ and $B$ and the consumption of children is obtained as a result of the following set of programs:

$$
\left\{\begin{array}{l}
\operatorname{Max}_{x^{A}} U^{A}\left(x^{A}\right) / e^{\prime} x^{A}=\alpha \\
\operatorname{Max}_{x^{B}} U^{B}\left(x^{B}\right) / e^{\prime} x^{B}=\beta \\
\operatorname{Max}_{x^{C}} U^{C}\left(x^{C}\right) / e^{\prime} x^{C}=y-\alpha-\beta .
\end{array}\right.
$$

Of course both $\alpha$ and $\beta$ depend on the internal household weight factor, $\mu$, and total income, $y$. The new sharing rule thus is bi-dimensional and writes functionally:

$$
\alpha\left(y_{A}, y_{B}, y_{0}\right)=f\left[y, \mu\left(y_{A}, y_{B}, y_{0}\right)\right] ; \quad \beta\left(y_{A}, y_{B}, y_{0}\right)=g\left[y, \mu\left(y_{A}, y_{B}, y_{0}\right)\right] .
$$

The two components of this new sharing rule must thus behave in an analogous way in response to any change in the structure of the household income that would maintain total income constant. Indeed, they would both react in that case to the same change in the weighting function, $\mu$.

In line with the previous section, we now consider the aggregate consumption behavior of the household. For any good $i$ consumed by the two adults and the children, the consumption function may now be written as the following sum:

$$
x_{i}\left(y_{A}, y_{B}, y_{0}\right)=F_{i}^{A}[\alpha]+F_{i}^{B}[\beta]+F_{i}^{C}[y-\alpha-\beta],
$$

where the arguments of functions $\alpha$ and $\beta$ have been omitted for simplicity.

The questions we now ask are the following. (i) Is it possible to derive from (20) tests of the form (17) of individual preferences and/or the Paretoefficiency hypothesis? (ii) Is it possible to recover from the observation of aggregate household consumption behavior information on the bi-dimensional sharing rule $(\alpha, \beta)$, and consequently on how much do the children get of the overall consumption budget $y$ ?

It can easily be checked that, under the assumptions that the preferences of the two adults satisfy (17) and that the allocation of the family budget among them and the children is Pareto-efficient, the proportionality of marginal propensities to consume (12) still holds. This provides a simple test of the whole model.

Concerning the recovery of the sharing rules, we will now see that the identifying techniques used in the previous section may be easily generalized and that it is indeed possible to derive from the observation of aggregate consumption behavior both functions $\alpha(\mathrm{)})$ and $\beta()$, up to a constant. We show how this is possible before discussing the implications of this result.

\subsection{Recovering the sharing rules}

(i) A trivial case: three exclusive goods. Let us begin with a simple case where its is assumed that good $i$ is consumed solely by member $A$, good 2 solely by 
member $B$ and good 3 solely by the children. We have then the following set of functional equations:

$$
\begin{aligned}
& x_{1}\left(y_{A}, y_{B}, y_{0}\right)=F_{1}^{A}\left[\alpha\left(y_{A}, y_{B}, y_{0}\right)\right] \\
& x_{2}\left(y_{A}, y_{B}, y_{0}\right)=F_{1}^{B}\left[\beta\left(y_{A}, y_{B}, y_{0}\right)\right] \\
& x_{3}\left(y_{A}, y_{B}, y_{0}\right)=F_{1}^{C}\left[y-\alpha\left(y_{A}, y_{B}, y_{0}\right)-\beta\left(y_{A}, y_{B}, y_{0}\right)\right] .
\end{aligned}
$$

Using the same method as in the preceding section, invert the first and second equations to get $\alpha=a\left(x_{1}\right)$ and $\beta=b\left(x_{2}\right)$ and substitute in the third to obtain the $\left(x_{1}, x_{2}\right)$-conditional demand function for $x_{3}$ :

$$
X_{3}=\theta_{3}\left(x_{1}, x_{2}, y\right)=F_{1}^{C}\left[y-a\left(x_{1}\right)-b\left(x_{2}\right)\right] .
$$

Denote by $\theta_{3 y}, \theta_{31}, \theta_{32}$ respectively the derivatives of this conditional demand function with respect to $y, x_{1}, x_{2}$. Taking ratios permits then identifying unambiguously $a^{\prime}\left(x_{1}\right)=\theta_{31} / \theta_{3 y}$ and $b^{\prime}\left(x_{2}\right)=\theta_{32} / \theta_{3 y}$ and thus the marginal propensities to consume good 1 by member $\mathrm{A}$ and good 2 by member 2 . The consumptions functions $F_{1}^{A}$ and $F_{2}^{B}$ are thus known up to a constant. $\alpha()$ and $\beta($ ) can then be recovered up to a constant from the first two equations in the system above. (Of course the observed demand functions must satisfy restrictions (12) for all this to be possible.)

(ii) A more interesting case: the triangular case. It is rather unlikely that three exclusive goods consumed respectively by the two adults and children in the household be observed. Interestingly enough, it is sufficient that only one such a exclusive good be observed for identification to be possible. In what follows, we consider the 'triangular' case where good 1 is consumed only by member $A$, good 2 is consumed by both members $A$ and $B$ but not by the children -i.e. this is a non-exclusive adult good- and good 3 is consumed by all family members. We thus have now:

$$
\begin{aligned}
& x_{1}\left(y_{A}, y_{B}, y_{0}\right)=F_{1}^{A}[\alpha] \\
& x_{2}\left(y_{A}, y_{B}, y_{0}\right)=F_{2}^{A}[\alpha]+F_{2}^{B}[\beta] \\
& x_{3}\left(y_{A}, y_{B}, y_{0}\right)=F_{3}^{A}[\alpha]+F_{3}^{B}[\beta]+F_{3}^{C}[y-\alpha-\beta] .
\end{aligned}
$$

Using the same kind of argument as in the previous case, and in particular different types of conditional demand functions, it is shown in the appendix that, provided that the aggregate demand functions satisfy the proportionality conditions (12), it is possible to recover the sharing rule from the above system, that is the functions $\alpha\left(y_{A}, y_{B}, y_{0}\right), \beta\left(y_{A}, y_{B}, y_{0}\right)$, up to a constant. However, a difference with the case examined in the previous section is that identification may require now using some third-order properties of demand functions, that is the way marginal propensities to consume out of the various income sources and changes in these marginal propensities depend themselves on income sources.

(iii) General case. Other situations comparable to the previous one or even slightly more complicated may be handled in the same manner. In par- 
ticular, methods analogous to the one shown in the appendix permit the partial identification of the sharing rules $\alpha\left(y_{A}, y_{B}, y_{0}\right), \beta\left(y_{A}, y_{B}, y_{0}\right)$, in presence of a child good and two adult goods, or even two adult goods and a good consumed by all. The proof is not given here for lack of space. It may also be expected that the same type of generalization as in the case with no children may apply. In other words, it should be possible to identify the sharing rule up to a constant even when the consumption of no exclusive good is observed. This would permit breaking with the Rothbarth approach implicit in the various situations we just described where identification is again essentially based on the distinction between adult or children goods and goods consumed by all household members.

\subsection{Implications}

That sharing rules may be identified up to a constant means that it is possible to know how changes in the budget constraint of a household, including the relative importance of the various income sources, will produce changes in the consumption budget of the various members of the family. By conditioning the analysis on various socio-demographic variables it is also possible to know how these marginal intra-family reallocations may depend on the characteristics of the household, and in particular its actual composition.

Knowing in what proportion an income subsidy given to a family with numerous children will be actually spent on children is of obvious practical importance. From that point of view, the collective approach to intra-family decisions permits avoiding restrictive assumptions of the type found in Rothbarth-like methods where identification proceeds exclusively by examining how the consumption of an adult good changes with income in families with and without children. Identification in the present case relies on less stringent assumptions. In particular, it does not rely on the comparison of consumption behavior across different demographic groups of households, and takes explicitly into account that many goods in a family are actually consumed by all members. This is essentially permitted by the assumption that aggregate consumption behavior responds differently to changes in the various income sources of the households and that this behavior must satisfy the general separability conditions summarized by equation (16).

It is the case, however, that identifying the sharing rule up to a constant means that, unlike in Rothbarth-like methods, nothing is known of the reference situation, that is, the actual allocation of the consumption budget between the various members of a family with given socio-demographic characteristics and income structure. From that point of view, it must be admitted that, if it were to lead to satisfactory empirical estimates, the collective approach to family consumption behavior could achieve only a limited progress in the identification of the cost of children.

\section{Conclusion}

All this brings us back to the apparently inescapable conclusion that identifying the cost of children, or in the present framework, the absolute -rather than differential- allocation of the consumption budget between family members 
requires some assumption of 'demographic separability' of consumption behavior. (see Deaton et al. 1989). In other words, identification can only be made by assuming that some individuals living in households with some given composition -e.g. married, married with children - have the same consumption behavior for some groups of goods as individuals with identical characteristics living in households with different composition, e.g. singles, married without children.

As mentioned above, this kind of assumption is at the heart of the Rothbarth approach to equivalence scales and the measurement of the cost of children. No use has been made of it in the collective approach yet, and it is certainly an interesting result of that approach that something, even partial, can be said of the intra-household allocation process without any assumption of demographic separability. The important point is that, unlike with the Rothbarth model, assuming that individual preferences for some goods are fully identical, whatever the consumption of these goods, for two persons living in families with different compositions is too much. How some limited demographic separability may be introduced in the collective approach so as to identify the constant in the sharing rule remains to be explored together with the empirical relevance of the method developed in the last part of this paper.

\section{Appendix}

\section{Identification of the twofold sharing rule in the triangular case}

Invert the first two equations of $\left(\mathrm{S}^{\prime}\right)$ to get $\alpha=a\left(x_{1}\right)$ and $\beta=b\left(x_{1}, x_{2}\right)$. Identifying the sharing rule is equivalent to identifying these functions $a()$ and $b()$. Substitute them in the third equation of $\left(\mathbf{S}^{\prime}\right)$ to get the following expression for the $\left(x_{1}, x_{2}\right)$-conditional demand for good 3 .

$$
X_{3}=\theta_{3}\left(y, x_{1}, x_{2}\right)=F_{3}^{A}(a)+F_{3}^{B}(b)+F_{3}^{C}(y-a-b) .
$$

Taking derivatives with respect to the three arguments of $\theta_{3}$ yields:

$$
\theta_{3 y}=F_{3}^{C^{\prime}} ; \theta_{32}=\left[F_{3}^{B^{\prime}}-F_{3}^{C^{\prime}}\right] b_{2} ; \theta_{31}=F_{3}^{A^{\prime}} \cdot a_{1}+F_{3}^{B^{\prime}} \cdot b_{1}+F_{3}^{C^{\prime}} \cdot\left(a_{1}+b_{1}\right),
$$

where $a_{1}$ is the derivative of a with respect to $x_{1}$ and $b_{i}$ the derivative of $b$ with respect to $x_{i}(i=1,2)$.

Taking the derivative again of the first equation in (A2) with respect to $x_{2}$ and $y$ permits identifying $b_{2}$ :

$$
b_{2}=\theta_{3 y 2} / \theta_{3 y y} \text {. }
$$

Substituting in the second equation of (A2) and making use of the first one yields the following expression for $F_{3}^{B^{\prime}}$ :

$$
F_{3}^{B^{\prime}}\left[b\left(x_{1}, x_{2}\right)\right]=\theta_{32} \cdot \frac{\theta_{3 y y}}{\theta_{3 y 2}}+\theta_{3 y} .
$$

Differentiating in turn this expression with respect to $x_{1}$ and $x_{2}$ and taking the 
ratio of these two derivatives yields $b_{1} / b_{2}$ as a function of the $1^{\text {st }}, 2^{\text {nd }}$ and $3^{\text {rd }}$ derivatives of the conditional demand function $\theta_{3}($ ). Together with (A3) this permits identifying $b_{1}$ so that the function $b()$ is known up to a constant.

To find $a_{1}$, we take the derivative of the first equation in (A2) with respect to $x_{1}$ and $y$. This yields:

$$
a_{1}+b_{1}=\theta_{3 y 1} / \theta_{3 y y} .
$$

Knowing $b_{1}$, it is then easy to get $a_{1}$.

\section{Endnotes}

1 These issues do not arise within an unitary view of the household since the intra-household allocation process is handled as a black box. We noted that the presence of public goods complicates substantially the Rothbarth model.

2 For the definition and use of 'conditional' demand functions, see Browning and Meghir (1991). Normally, this conditional demand should depend on two out of the three arguments of the original demand functions, the third one being replaced by the conditioning on $x_{1}$. As may be seen in (16) an implication of the collective model is that it actually depends only on total income and the conditioning demand.

${ }^{3}$ Of course, it is necessary that conditions (12) above be satisfied on the observed consumption functions of $x_{1}$ and $x_{2}$ in order for these derivations to be possible.

${ }^{4}$ Of course, this assumption becomes more debatable in presence of children, known as the main explanatory factor of inactivity for women.

\section{References}

Barten A-P (1964) Family Composition, Prices and Expenditure Patterns. In: Hart P-E, Mills G, Whitaker J-K (eds) Econometric Analyses for National Economic Planning. Butterworth: London

Blundell R, Lewbel A (1991) The Information Content of Equivalence Scales. Journal of Econometrics 50(12):49-68

Bourguignon F, Chiappori P-A (1992) Collective Models of Household Behavior: An Introduction. European Economic Review 36(23):355-364

Bourguignon F, Browning M, Chiappori P-A (1993) Intra Household Allocation of Consumption: A Model and Some Evidence from French Data. Annales d'Economie et de Statistique 29:137-156

Browning M, Bourguignon F, Chiappori P-A, Lechêne V (1994) Income and Outcomes: A Structural Model of Intrahousehold Allocation. Journal of Political Economy 102(6):1067-1096

Browning M, Meghir C (1991) The Effects of Male and Female Labor Supply on Commodity Demands. Econometrica 59(4):925-951

Chiappori P-A (1988) Rational Household Labor Supply. Econometrica 56(1):63-90

Chiappori P-A (1992) Collective Labor Supply and Welfare. Journal of Political Economy $100(3): 437-467$

Deaton A, Muellbauer J (1986) On Measuring Child Costs: With Applications to Poor Countries. Journal of Political Economy 94(4):720-744

Deaton A, Ruiz Castillo J, Thomas D (1989) The Influence of Household Composition on Household Expenditure Patterns: Theory and Spanish Evidence. Journal of Political Economy 97(1):179-200

Fisher F (1987) Household Equivalence Scales and Interpersonal Comparisons. Review of Economic Studies 54(3):519-524

Folbre N (1994) Children as Public Goods. American Economic Review 84(2):86-90

Gronau R (1991) The Intrafamily Allocation of Goods-How to Separate the Adult from the Child. Journal of Labor Economics 9(3):207-235 
Kapteyn A (1994) The Measurement of Household Cost Functions: Revealed Preference versus Subjective Measures. Journal of Population Economics 7(4):333-350

Lazear E, Michael R (1986) Estimating the Personal Distribution of Income with Adjustment for Within-Family Variation. Journal of Labor Economics 4(3):S216-S239

Pollak R, Wales T (1979) Welfare Comparisons and Equivalence Scales. American Economic Review 69(2):216-221

van Praag B, Warmaar M (1997) The Cost of Children and the Use of Demographic Variables in Consumer Demand. In: Rosenzweig M, Stark O (eds) Handbook of Population and Family Economics. Elsevier: Amsterdam, 241-273

Weiss Y, Willis R (1985) Children as Collective Goods and Divorce Settlements. Journal of Labor Economics 3(3):268-292 